\title{
Survival Outcomes in Male Breast Cancer - A Single Institution Experience
}

\author{
Nisha Hariharan
}

Basavatarakam Indo American Cancer Hospital \& Research Institute, Hyderabad, India.

\begin{abstract}
Introduction: Male breast cancer is an uncommon entity. Due to the scarce numbers, treatment protocols have largely been extrapolated from available evidence for female breast cancers. Methods: We analysed the clinicopathological features and survival outcomes for male breast cancer patients treated at our institute between January 2010 and June 2016. Results: Of the 5534 women treated at our institute, we screened 40 male breast cancers of whom 33 had available follow up data and were included in the present analysis. Male breast cancer constituted $0.7 \%$ of all breast cancers. The median age was 60 years and the median tumor size was $3 \mathrm{~cm}$ with $66 \%$ of patients having nodal disease at presentation. Invasive ductal carcinoma was the most common histology and $97 \%$ were hormone receptor positive. Most of the patients $(87.8 \%)$ underwent an upfront modified radical mastectomy. With a median follow up of 36 months, 10 patients experienced recurrences all of which were distant metastasis ( 3 to the bone, 1 to the brain, and 6 had visceral metastasis). Of these, 7 patients succumbed to the disease. The 3-year overall survival was $78.7 \%$. Conclusion: Male breast cancer is a rare clinical entity and current treatment guidelines follow those for women. Due to the lack of awareness, men often present to clinics at an advanced stage. Social support targeted at improving awareness and access to treatment could improve outcomes in this cohort.
\end{abstract}

Keywords: Male breast cancer- survival

Asian Pac J Cancer Care, 3 (4), 71-74

\section{Introduction}

Male breast cancer is a rare entity comprising less than $1 \%$ of all breast cancers (1). The American Cancer Society estimated that about 2600 new male breast cancers would be diagnosed in the United States with a projected 440 deaths amongst them in the year 2016 (2).

Evidence in male breast cancer is sparse and limited to retrospective audits and case series reports. The classification and management has been extrapolated from available randomised clinical trials for female breast cancers. However, there are noticeable differences in the presentation of breast cancer in men and women. Literature suggests that men are more likely to present at an advanced stage with significantly higher nodal involvement (3). It is plausible this could be due to the lack of awareness that breast cancer can afflict men. Consequently, there is often a paucity of social support for these patients.
Submission Date: 08/02/2018 Acceptance Date: 10/05/2018

In our study, we attempted to evaluate the clinicopathological characteristics and survival outcomes for male breast cancer patients treated at our institute.

\section{Materials and Methods}

All male patients presenting to the Breast Oncology department at our institute between 2010 and 2016 were screened. All newly diagnosed breast cancer cases with available receptor status were included in the analysis. Source files in the Medical Records and the Electronic Medical Records were screened to retrieve data. Demographic data, tumour characteristics and treatment related details have been documented.

Staging was assessed according to the American Joint Committee on cancer criteria (7th edition). The estrogen receptor (ER) and progesterone receptor (PR) results were screened and interpreted as positive when more

\section{Corresponding Author:}

Dr. Nisha Hariharan

Basavatarakam Indo American Cancer Hospital \& Research Institute, Hyderabad, India.

Email: dr.nishahariharan@gmail.com 
than $1 \%$ of tumour cells showed positive nuclear staining. For the HER2 status, a strong and complete membrane staining in more than $30 \%$ of tumour cells was considered as a positive immunostaining (score $3+$ ). Data were summarised as frequencies and proportions. Patients were contacted telephonically if their last hospital visit was more than 6 months earlier. Overall survival was calculated until death from any cause or last contact for living patients. An Institutional Ethics Committee approval was obtained for the study.

\section{Results}

Of the 5534 patients who underwent surgery for breast tumours at our institute from January 2010 to June 2016, 51 were male patients. Among them, 11 had gynaecomastia or benign adenosis. 40 male patients presented with breast carcinoma, of whom, 33 had available receptor status and follow up data, and were included in the analysis. The median follow-up was 36 months.
The median age at presentation was 60 years and the median tumour size was $3 \mathrm{~cm} .39 \%$ of the patients presented at an advanced stage and $66 \%$ had nodal disease at presentation. Only one patient had bilateral carcinoma. The predominant histology was invasive ductal carcinoma. None of the patients had carcinoma in situ. $18 \%$ had grade 3 tumours. Hormone receptor positive status (ER and/or PR) was seen in $97 \%$ of the cohort. Her 2 results were available for 13 patients, of whom 3 tested positive. The cohort demographics are shown in Table 1.

29 patients underwent an upfront modified radical mastectomy of whom 2 had involvement of skin or nipple areolar complex. In the adjuvant setting, 12 patients received Adriamycin and Taxol based regimens and 18 received adjuvant radiation (Table 2 ).

With a median follow up of 3 years, 10 men in our cohort had a disease recurrence. All of these patients had distant metastasis (Table 3 ). There were no isolated local recurrences. 7 patients died of disease recurrence during the follow up. The 3 -year overall survival in

Table 1. Cohort Demographics

\begin{tabular}{lcc}
\hline Demographics & Groups & Frequency $(\%)$ \\
\hline Clinical stage & Operable cancer & $20(60.6 \%)$ \\
Bilaterality & Locally advanced cancer & $13(39.3 \%)$ \\
Tumor size & No & $32(96.9 \%)$ \\
& Yes & $1(3.03 \%)$ \\
Nodal status & $<2 \mathrm{~cm}$ & $3(9.09 \%)$ \\
& $2-5 \mathrm{~cm}$ & $25(75.7 \%)$ \\
Histology & $>5 \mathrm{~cm}$ & $5(15.1 \%)$ \\
& Negative & $11(33.3 \%)$ \\
Grade & $1-3$ nodes & $9(27.2 \%)$ \\
& $>=4$ nodes & $13(39.3 \%)$ \\
Lymphovascular invasion & Duct carcinoma & $33(100 \%)$ \\
Hormone receptor status & Others & 0 \\
& Grade 1 & $2(6.06 \%)$ \\
& Grade 2 & $25(75.7 \%)$ \\
& Grade 3 & $6(18.1 \%)$ \\
& No & $11(3.03 \%)$
\end{tabular}

Table 2. Treatment Details

\begin{tabular}{lcc}
\hline Treatment details & No of patients \\
\hline Primary treatment & Upfront surgery & 29 \\
& Neoadjuvant chemotherapy & 4 \\
Chemotherapy schedules & Anthracyclines alone & 21 \\
& Anthracyclines+ Taxanes & 12 \\
Adjuvant radiation & Taken & 18 \\
\hline
\end{tabular}


Table 3. Site of First Recurrence

\begin{tabular}{lc}
\hline Site of recurrence & No of patients \\
\hline Isolated local recurrence & 0 \\
Lung/Liver & 6 \\
Bone & 3 \\
Brain & 1 \\
\hline
\end{tabular}

the cohort was $78.7 \%$.

\section{Discussion}

Male breast cancer is a relatively rare occurrence. Literature suggests that it forms less than one percent of all breast cancers (3). In our cohort too, it constituted $0.7 \%$ of all the patients treated at our institute.

The median age at presentation among the 5534 women treated during the same time period at our institute is 51 years. Among male breast cancers, the median age at presentation was a decade later, with $39 \%$ of the men presenting with locally advanced disease. In a similar analysis by Gogia et al (4), comprising of 76 male breast cancers registered over a period of 17 years, they observed the median age at presentation to be 59 years with $59.3 \%$ of the patients presenting at an advanced stage. Another study from north India (5), documented a median age of 56 years with $43.3 \%$ presenting with advanced disease. In a large review of the Surveillance, Epidemiology and End Results (SEER) database, including 2537 men with breast cancer, Giordano et al (3) observed that the median age at presentation was 67 years compared to 62 years among women treated at the same time. However, a majority of the male patients in this review presented with early breast cancer (78.4\%). Data from Indian cohorts concur with the West that male breast cancer presents a few years later than in women. However, we still continue to suffer from a late stage at presentation among men.

All the patients in our cohort had invasive ductal carcinoma (IDC) with $97 \%$ of them being ER/PR positive. Shah et al (6), in their review of 42 male breast cancers found $83.3 \%$ to have IDC and $89.7 \%$ to be hormone receptor positive. Likewise, D Ram et al (7) in their analysis of 27 men, observed that $85.2 \%$ of them presented with IDC and $77.8 \%$ with hormone positive tumors. In an analysis of 2992 male patients from the SEER program, Leone et al (8).

Documented an $85 \%$ ductal carcinoma incidence with a $95.1 \%$ ER receptor positivity. Male breast carcinoma resembles carcinoma in women with respect to predominant ductal histology. Also, most carcinomas in men are hormone receptor positive, allowing for the use of tamoxifen in the adjuvant setting.

With a median follow up of 3 years in our cohort, 10 patients experienced a disease recurrence and 7 of them succumbed to the disease. Shukla et al (9), in their review of 42 male patients from a regional cancer centre in north India, found the actuarial overall survival and disease-free survival to be $91.7 \%$ and $66.7 \%$ respectively, at 4 years. In a retrospective survey of male breast cancer from four French institutions, from 2000 to 2010, Oger and colleagues (10), documented an overall survival of $79.2 \%$ at 5 years and $70.8 \%$ at 10 years. They observed that age, tumor size and histological capsular rupture are factors that significantly influence overall survival.

In conclusion, male breast cancer is a rare clinical entity and forms less than one percent of all breast cancers. The median age at presentation is a decade later than in women. Due the lack of awareness, men often present to clinics at an advanced stage. Most of the patients are amenable to upfront surgery followed by adjuvant therapy. A majority of the tumors are hormone receptor positive.

Social programs aimed at improving awareness could help in detecting this cancer at an early stage.

\section{Funding}

This research did not receive any specific grant from funding agencies in the public, commercial, or not-forprofit sectors.

\section{Ethics}

An institutional Ethics Committee approval was obtained.

\section{Acknowledgements}

We would like to extend our thanks to Yashwanth Sankranti (ex- clinical trial co-ordinator at Basavatarakam Indo American Cancer Hospial \& Research Institute, India) for his help with the data.

\section{References}

1. Ian S Fentiman, Alain Fourquet, Gabriel N Hortobagyi, Male breast cancer, The Lancet, Volume 367, Issue 9510, 2006, Pages 595-604, ISSN 0140-6736, http://dx.doi.org/10.1016/ S0140-6736(06)68226-3.

2. Siegel, R. L., Miller, K. D. and Jemal, A. (2016), Cancer statistics, 2016. CA: A Cancer Journal for Clinicians, 66: 7-30. doi:10.3322/caac.21332

3. Giordano, S. H., Cohen, D. S., Buzdar, A. U., Perkins, G. and Hortobagyi, G. N. (2004), Breast carcinoma in men. Cancer, 101: 51-57. doi:10.1002/cncr.20312

4. Gogia A, Raina V, Deo S, Shukla N K, Mohanti B K. Male breast cancer: A single institute experience. Indian J Cancer 2015;52:526-9

5. Rai, B. et al. "Breast cancer in males: a PGIMER experience." Journal of cancer research and therapeutics 11 (2005): 31-3.

6. Shah S, Bhattacharyya S, Gupta A, Ghosh A, Basak S. Male breast cancer: a clinicopathological study of 42 patients in eastern India. Indian journal of surgical oncology. 2012;3(3):245-9

7. Ram D, K R Suhas, Selvakumar V P, Shukla H, Goel A, Kumar R \& Kumar K. (2017). Male breast cancer: A retrospective review of clinical profile from a tertiary cancer care center of India. South Asian Journal of Cancer. 6. 141. 10.4103/sajc.sajc_2_17.

8. Leone, J.P, Zwenger, A.O, Iturbe, J. Et al Prognostic factors in male breast cancer: a population-based study. Breast Cancer Res Treat (2016) 156: 539 https://doi.org/10.1007/ s10549-016-3768-1

9. Shukla NK, Seenu V, Goel AK, Raina V, Rath GK, Singh R, et al. Male breast cancer: A retrospective study from a regional 
cancer center in Northern India. J Surg Oncol 1996;61:143-8

10. Oger AS, Boukerrou M, Cutuli B, Campion L, Rousseau E, Bussières E, Raro P, Classe JM. Male breast cancer: prognostic factors, diagnosis and treatment: a multiinstitutional survey of 95 cases. Gynecol Obstet Fertil. 2015 Apr;43(4) 290-296. doi:10.1016/j.gyobfe.2015.02.010. PMID: 25818033.

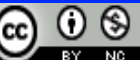

This work is licensed under a Creative Commons AttributionNon Commercial 4.0 International License. 